\title{
EFICIENCIA REPRODUCTIVA DE LAS ANGIOSPERMAS DE UN BOSQUE INUNDABLE EN LA CUENCA DEL LAGO DE MARACAIBO (VENEZUELA)
}

\author{
Reproductive efficiency of the angiosperms of a seasonally flooded forest in the lake \\ Maracaibo basin (Venezuela)
}

YENI BARRIOS ${ }^{1}$ \& \& NELSON RAMÍREZ²]

\begin{abstract}
Resumen: La relación fruto/flor, relación semilla/óvulo y fecundidad relativa fueron evaluadas en 25 especies de un bosque inundable en la cuenca del lago de Maracaibo (Venezuela) y correlacionadas con el número y costo de las estructuras reproductivas. Estos estimadores, junto con la biomasa asignada a frutos y semillas y la relación pericarpio/semilla, fueron analizados entre formas de vida, sistemas reproductivos y síndromes de dispersión de semillas. En general, la relación fruto/flor y la fecundidad relativa fueron bajas mientras que la relación semilla/óvulo fue alta. La relación fruto/flor estuvo asociada negativamente con el número de flores por inflorescencia, y al igual que la fecundidad relativa, con el peso del fruto y de la semilla. La relación semilla/óvulo estuvo negativamente relacionada con el número de óvulos por flor y con el peso del fruto. La relación fruto/flor y la fecundidad relativa fueron mayores en especies herbáceas, autógamas y/o de dispersión abiótica que en sus contrapartes leñosas, xenógamas y/o zoocoras las cuales presentaron mayor peso semilla, y en el caso de las especies xenógamas, también mayor relación pericarpio/semilla que las autógamas. La relación semilla/óvulo fue mayor en especies de dispersión abiótica que en las zoocoras. Estos resultados sugieren una fuerte influencia del número y costo de las estructuras reproductivas en la eficiencia reproductiva de las especies.
\end{abstract}

Palabras clave: Fecundidad relativa, humedales, producción de frutos y semillas, relación fruto/ flor, relación semilla/óvulo.

Summary: Fruit/flower ratio, seed/ovule ratio and relative fecundity were evaluated on 25 species of a seasonally flooded forest in the lake Maracaibo basin and correlated with number and cost of reproductive structures. These estimators, together with the biomass assigned to fruits and seeds and the pericarp/seed ratio, were analyzed between life forms, reproductive systems and seed dispersal syndromes. In general, fruit/flower ratio and relative fecundity were low, but seed/ovule ratio was high. Fruit/flower ratio was negatively associated to the number of flowers per inflorescence, and like the relative fecundity, to fruit and seed weight. Seed/ovule ratio was negatively associated to the number of ovules per flower and to fruit weight. Fruit/flower ratio and relative fecundity were higher in herbaceous, autogamous and/or abiotically dispersed species than in woody, xenogamous and/or zoochorus species which showed higher seed weight, and regarding xenogamous species also higher pericarp/seed ratio than the autogamous ones. Seed/ovule ratio was higher in abiotically dispersed species than in the zoochorus ones. These results suggest a strong influence of number and cost of reproductive structures in species reproductive efficiency.

Key words: Fruit and seed set, fruit/flower ratio, relative fecundity, seed/ovule ratio, wetlands.

${ }^{1}$ Universidad de Los Andes, Facultad de Ciencias, Instituto Jardín Botánico de Mérida, Apdo. 52, Mérida 5212, Venezuela. E-mail: yeniceleste@gmail.com

${ }^{2}$ Universidad Central de Venezuela, Facultad de Ciencias, Instituto de Biología Experimental, Centro de Botánica Tropical, Apdo. 48312, Caracas 1041A, Venezuela. E-mail: nramirez220252@gmail.com 


\section{Introducción}

La producción de frutos y semillas en las angiospermas está limitada por muchos factores, las hipótesis basadas en la aptitud femenina señalan entre ellos a la baja disponibilidad de polen, la sobreproducción deflores comoatracción y/o compensación ante la impredecibilidad del ambiente, y el costo de las estructuras reproductivas (Stephenson, 1981; Bawa et Weeb, 1984; Sutherland et Delph, 1984; Galen et al., 1985; Sutherland, 1986; Fenster, 1991; Ramírez, 1992, 1993; Ramírez et Berry, 1993; Burd, 1994, 2004, 2009; Ashman et al., 2004; Harder et al., 2008). La hipótesis de donación de polen, por otra parte, plantea que existe una sobreproducción de flores en las especies hermafroditas que contribuye únicamente a aumentar la aptitud masculina de la planta y que a esto se debe la menor proporción de frutos que producen en comparación con sus contrapartes monoicas y dioicas (Sutherland et Delph, 1984). En estos sistemas sexuales (monoecía y dioecía), la separación de las funciones sexuales y el ajuste en el número de flores femeninas y masculinas aumenta la relación fruto/flor (Sutherland et Delph, 1984; Sutherland, 1986) y en consecuencia la eficiencia reproductiva de las especies.

Una excepción a la baja producción de frutos predicha en las especies hermafroditas está relacionada con los sistemas reproductivos. En este sentido, la hipótesis de donación de polen plantea que las especies autógamas muestran una producción de frutos mayor que sus contrapartes xenógamas como resultado de la menor asignación a la función masculina que se deriva de la autopolinización (Sutherland et Delph, 1984; Sutherland, 1986; Charlesworth et Charlesworth, 1987; Brunet, 1992). En concordancia con esta idea, la hipótesis de autoincompatibilidad enfatiza que la producción de frutos en las especies xenógamas autoincompatibles, además de estar limitada por la menor asignación a la función femenina, está limitada por la reacción de autoincompatibilidad en los estigmas la cual puede impedir la germinación del polen exógeno (Sutherland et Delph, 1984; Sutherland, 1986). Estas condiciones, aunadas a la baja predecibilidad de la polinización cruzada (Burd, 1994; Ashman et al., 2004), aumentan la brecha entre la eficiencia reproductiva de especies xenógamas y autógamas, aunque la pérdida de variabilidad genética en estas últimas también puede generar depresión por endogamia (Charlesworth et Charlesworth, 1987; Fenster, 1991; Husband et Schemske, 1996).

Otro de los factores que ha sido relacionado con la eficiencia reproductiva de las especies de plantas es la forma de vida (Sutherland, 1986; Ramírez, 1993). En este sentido, la relación fruto/flor suele ser significativamente menor en las formas de vida leñosas que en las herbáceas (Sutherland, 1986; Ramírez, 1993). Entre las hipótesis que han sido planteadas para explicar estos patrones, se encuentran las diferencias temporales entre las fenofases de floración y fructificación en ambos grupos de especies y el efecto del costo energético de los frutos (Ramírez, 1993). Las especies leñosas frecuentemente producen frutos costosos que requieren un largo período de fructificación y están sujetos a las variaciones temporales en la disponibilidad de recursos. Las especies herbáceas, en cambio, producen frutos económicos madurados en corto tiempo, estas últimas pueden ajustar la producción de flores a la cantidad de recursos disponibles aumentando la predecibilidad de la producción de frutos con relación a las especies leñosas (Sutherland, 1986; Ramírez, 1993).

El síndrome de dispersión de semillas, por su correlación con el costo de las estructuras reproductivas, también ha sido relacionado con la eficiencia en la producción de frutos y semillas. Ramírez et Berry (1993) señalan que las especies epizoocoras y mirmecocoras muestran los menores niveles de frutos abortados y los mayores niveles de semillas producidas en asociación con bajos valores de biomasa asignada en frutos y semillas. Las especies mamalocoras, en cambio, presentan los mayores niveles de aborto de frutos y los menores niveles de producción de semillas asociados a una alta asignación de biomasa en frutos y semillas (Ramírez et Berry, 1993).

En este trabajo se evaluó la eficiencia en la producción de frutos y semillas de las especies de una comunidad inundable en la cuenca del lago de Maracaibo (Venezuela) y su relación con las variables: número y costo de las estructuras reproductivas, forma de vida, sistema reproductivo y síndrome de dispersión de semillas. Las preguntas que se formularon 
fueron las siguientes: 1. ¿La producción de frutos y semillas varía entre clases de forma de vida, sistema reproductivo y/o síndrome de dispersión de semillas?; 2. ¿Varía la biomasa asignada a frutos y semillas entre clases de forma de vida, sistema reproductivo y/o síndrome de dispersión de semillas?; 3. ¿Está relacionada la eficiencia reproductiva con el número de flores por inflorescencia, de óvulos por flor y/o con la biomasa asignada a frutos y semillas?; y 4. ¿Cuáles podrían ser las hipótesis explicativas de la producción de frutos y semillas en comunidades inundables?

\section{Materiales y Métodos}

\section{Área de estudio}

El estudio fue realizado en un bosque seco ralo $(\approx 81$ ha) ubicado en la planicie de inundación del río Mene, costa oriental del lago de Maracaibo, Venezuela (10²6'46'" - 10²7'28,8' N,

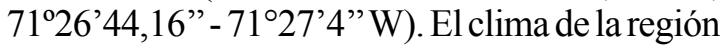
es semiárido (BSh) (Peel et al., 2007), los datos de la estación climatológica más cercana $(\approx 1 \mathrm{~km}$ del área de estudio) indican que la precipitación media anual es $570 \mathrm{~mm}$, la evapotranspiración media anual es $2431 \mathrm{~mm}$ y la temperatura media mensual es $29^{\circ} \mathrm{C}$. Las precipitaciones se distribuyen entre abril y noviembre, período en el que el bosque permanece anegado o al menos con suelo saturado de agua debido al desborde del río Mene. La estación seca comprende de diciembre a marzo y durante este período la mayor parte del bosque presenta suelo seco.

El inventario florístico del lugar incluye 118 especies pertenecientes a 41 familias de angiospermas según APG IV (2016). La distribución de las especies a nivel de formas de vida es $42,7 \%$ hierbas, $25,6 \%$ árboles, $12,8 \%$ arbustos, 6,8\% lianas leñosas, 6,8\% lianas herbáceas, $4,2 \%$ sufrútices y $0,8 \%$ epífitas. El $28 \%$ de las especies registradas son plantas de humedales (hidrófitas y helófitas según Velásquez, 1994), principalmente hierbas (91\%), seguidas de sufrútices (6\%) y arbustos $(3 \%)$ (Barrios, datos no publ.).

La vegetación presenta dos estratos: uno superior, discontinuo, de 8-20 m de alto, en el que predominan Copernicia tectorum (Kunth) Mart., Cynophalla hastata (Jacq.) J. Presl,
Coccoloba caracasana Meisn., Cordia dentata Poir., Geoffroea spinosa Jacq., Pithecellobium lanceolatum (Humb. \& Bonpl. ex Willd.) Benth., Guazuma ulmifolia Lam. y Ruprechtia ramiflora (Jacq.) C. A. Mey., y uno inferior, de hasta $3 \mathrm{~m}$, dominado por Hecatostemon completus (Jacq.) Sleumer y Rosenbergiodendron formosum (Jacq.) Fagerl. var. formosum. Por otra parte, en áreas anegadas y con baja densidad de árboles prevalecen helófitas e hidrófitas, entre ellas Cyperus articulatus L., Eleocharis mutata (L.) Roem. \& Schult., Typha domingensis Pers., Nymphaea spp., Pistia stratiotes L. y varias especies de lemnoides (Barrios, 2017).

\section{Selección y clasificación de las especies}

Las 25 especies estudiadas (Anexo 1) fueron seleccionadas con base en el número de individuos disponibles en el área de estudio: $\approx 50$ individuos/ ha para las especies leñosas $\mathrm{y} \approx 10$ individuos/100 $\mathrm{m}^{2}$ para las especies herbáceas. Este criterio permitió contar con suficientes individuos por especie para determinar los sistemas sexuales, recolectar las estructuras reproductivas (flores, frutos) y realizar el seguimiento in situ de la producción de frutos y semillas. En este último aspecto, se procuró que los individuos estudiados, y/o las manchas en el caso de las hierbas, estuviesen distantes para minimizar el riesgo de describir una misma unidad genética. Por otra parte, también se intentó mantener en la muestra de especies la misma proporción de formas de vida y de plantas de humedales observada en la comunidad. Los ejemplares testigos fueron depositados en los herbarios HERZU y MERF.

Las especies fueron clasificadas según sus formas de vida como árboles, arbustos, trepadoras, sufrútices, hierbas y epífitas (Anexo 1) manteniendo el criterio de Ramírez (1993). Las clases de sistemas sexuales fueron establecidas de la siguiente manera: hermafroditismo, monoecia y dioecia (Anexo 1). Las especies hermafroditas se reconocieron por la presencia de flores bisexuales en todos los individuos, las especies monoicas por la presencia de flores unisexuales femeninas y masculinas en la misma inflorescencia o individuo, y las dioicas por la presencia de flores femeninas o masculinas por individuo.

Los sistemas reproductivos fueron inferidos con base en las siguientes características florales 
estudiadas por Barrios (2017) en la misma muestra de especies: 1) la relación polen/óvulo (P/O) (Cruden, 1977), 2) la coincidencia o no coincidencia temporal de la dehiscencia de las anteras con la receptividad estigmática (adicogamia o dicogamia), 3) la separación espacial entre anteras y estigmas (hercogamia) y 4) la producción de frutos en condiciones de exclusión de polinizadores (reproducción espontánea). Se consideraron autógamas, sin descartar apomixis, las especies que produjeron frutos de manera espontánea y/o que mostraron el conjunto de las siguientes características: adicogamia, ausencia de hercogamia y baja relación polen/óvulo $(\mathrm{P} / \mathrm{O} \leq 400$, cifra cercana al límite superior de los valores registrados por Cruden (1977) para plantas autógamas facultativas). Por otra parte, se consideraron xenógamas, las especies que no produjeron frutos de manera espontánea y/o que mostraron las siguientes características: dicogamia y/o hercogamia, y elevada relación polen/óvulo $(\mathrm{P} / \mathrm{O}>$ 400) (Cruden, 1977). En algunos casos, además, se utilizaron las referencias disponibles en la literatura sobre los sistemas de compatibilidad de las especies para complementar esta clasificación (Anexo 2).

Los síndromes de dispersión de semillas se definieron de acuerdo con el criterio de Van der Pijl (1979): 1) la anemocoria incluyó a las especies con diásporas pequeñas, livianas y/o con adaptaciones estructurales al vuelo (pelos, alas), 2) la hidrocoria incluyó a las especies con diásporas flotantes lo cual se determinó en condiciones de laboratorio, 3) la ornitocoria incluyó a las especies con diásporas carnosas atractivas para las aves, generalmente rojas, negras, blancas, azules o con una mezcla contrastante de estos colores, 4) la mamalocoria incluyó a las especies con diásporas carnosas atractivas para los murciélagos y mamíferos no voladores las cuales se reconocieron por la presencia de aromas fuertes y colores poco llamativos (Anexo 1).

\section{Biomasa asignada a frutos y semillas}

Para cada una de las especies se estimó el peso seco de frutos y semillas así como la relación pericarpio/semilla (P/S) (Anexo 3). El peso fue determinado por separado en 40 frutos y 40 semillas, de al menos diez individuos por especie, previamente secados en una estufa a $60^{\circ} \mathrm{C}$. La relación $\mathrm{P} / \mathrm{S}$ se estimó mediante la fórmula $\mathrm{P} /$ $\mathrm{S}=$ (peso promedio del fruto - peso promedio de semillas por fruto) / peso promedio de semillas por fruto. El peso promedio de semillas por fruto fue el producto del número promedio de semillas por fruto por el peso promedio de las semillas individuales. Esta relación $(\mathrm{P} / \mathrm{S})$ proporciona una medida relativa del costo de la inversión materna/ progenie (Ramírez et Berry, 1993).

\section{Eficiencia natural en la producción de frutos y semillas}

La eficiencia natural en la producción de frutos fue estimada cuantificando el número de flores con estructuras sexuales femeninas y el número de frutos formados en al menos 50 inflorescencias distribuidas en un mínimo de diez individuos por especie. En el caso de las especies con flores solitarias, se realizó el seguimiento de al menos 30 flores en antesis hasta la maduración de los frutos. La relación fruto/flor fue expresada comola relación entre el número promedio de frutos/infrutescencias y el número promedio de flores/inflorescencia, o en las especies con flores solitarias, como la relación entre el número de frutos formados y el número inicial de flores marcadas.

Para estimar la eficiencia en la producción de semillas se cuantificaron el número de semillas, incluidas las abortadas, en 100 frutos de al menos 10 individuos por especie. Se consideraron semillas abortadas, las semillas mal formadas, de tamaños reducidos, aplastadas o carentes de embrión. La relación semilla/óvulo se calculó dividiendo el número promedio de semillas por fruto entre el número promedio de óvulos por flor estimado en una muestra de cien flores de al menos diez individuos por especie. La fracción de semillas abortadas se calculó dividiendo el número de semillas abortadas por fruto entre el número promedio de semillas totales por fruto. La fecundidad relativa (FR), definida como la fracción de semillas sanas producidas por inflorescencia, fue determinada de acuerdo con la expresión propuesta por Ramírez (1992):

$\mathrm{FR}=\underline{(\text { Semillas totales/fruto }- \text { semillas abortadas/fruto }) \times \text { frutos/infrutescencia }}$ Óvulos/flor $\times$ flores/inflorescencia

Para efectos de esta expresión, el número promedio de frutos por infrutescencia y de flores 
por inflorescencia, en el caso de las especies con flores solitarias, fue sustituido por el número de frutos formados y el número inicial de flores evaluadas, respectivamente.

\section{Análisis estadísticos}

Dado el bajo tamaño de la muestra, para efecto de estos análisis fueron combinadas algunas categorías afines, por ejemplo, las especies herbáceas, sufruticosas y epífitas fueron reunidas en la categoría "herbáceas" y las especies arbóreas, arbustivas y trepadoras en la categoría "leñosas". Asimismo, se combinaron los síndromes de dispersión de semillas bióticos (ornitocoria, mamalocoria) y los abióticos (hidrocoria, anemocoria). En el caso de las especies diplocoras, es decir, con más de un síndrome de dispersión (Van der Pijl, 1972), la categoría asignada fue aquella que incluyera el síndrome de dispersión principal.

La relación entre las variables forma de vida, sistema reproductivo y síndrome de dispersión de semillas fue explorada mediante la prueba exacta de Fisher. La variabilidad de los estimadores de la eficiencia reproductiva natural (relación fruto/flor, relación semilla/ óvulo y fecundidad relativa) fue examinada a nivel de formas de vida (leñosas, herbáceas), sistemas reproductivos (xenogamia, autogamia) y síndromes de dispersión de semillas (bióticos, abióticos) mediante una prueba $t$. Los valores fueron previamente transformados por la fórmula $\operatorname{arcsen} \sqrt{ } \mathrm{p}$, donde $\mathrm{p}$ representa la proporción de fruto o semillas formados (Sokal et Rohlf, 1969). La variabilidad de la biomasa asignada a frutos y semillas, así como de la relación pericarpio/ semilla, fue igualmente analizada entre clases de formas de vida, sistemas reproductivos y síndromes de dispersión de semillas; no obstante, cuando los datos fueron heterocedásticos se utilizó la prueba de Welch (Hammer et al., 2001). Estos valores fueron previamente transformados por la ecuación $\sqrt{ }(X+1)$, donde $X$ es el valor de la variable (Sokal et Rohlf, 1969). El grado de asociación entre la eficiencia reproductiva y el número de flores por inflorescencia, de óvulos por flor y la biomasa asignada a frutos y semillas, así como entre el peso de frutos y semillas, fue determinado con el coeficiente de correlación de rango de Spearman (Rs). Todos los análisis fueron realizados con el programa PAST 3.0
(Hammer et al., 2001) y el nivel de significancia mínimo establecido fue igual a 0,05 .

\section{Resultados}

Eficiencia natural en la producción de frutos y semillas

La producción de frutos en las especies estudiadas fue baja en promedio, no obstante, mostró gran variabilidad (relación fruto/flor $\bar{X}$ $=0,34 \pm 0,35 ; n=23 \mathrm{spp}$.). La producción de semillas, en cambio, fue alta y menos variable (relación semilla/óvulo $\bar{X}=0,72 \pm 0,28 ; n=25$ spp.). Con relación a la calidad de las semillas, los frutos mostraron predominio de semillas sanas (fracción de semillas abortadas $\bar{X}=0,00$ $\pm 0,00 ; n=25$ spp.). No obstante, la tendencia en la producción de frutos afectó la proporción de semillas sanas producidas por inflorescencia ya que este resultado también fue bajo en promedio y altamente variable entre las especies (fecundidad relativa $\bar{X}=0,29 \pm 0,31 ; \mathrm{n}=23 \mathrm{spp}$.) (Tabla 1).

Relación entre las variables forma de vida, sistema reproductivo y sindrome de dispersión de semillas

La forma de vida estuvo significativamente relacionada con el sistema reproductivo (Fisher $p=0,0028)$ y con el síndrome de dispersión de semillas (Fisher $p=0,0001$ ): las especies leñosas fueron exclusivamente xenógamas y mostraron síndromes de dispersión mayormente bióticos, mientras que las especies herbáceas fueron tanto xenógamas como autógamas y mostraron síndromes de dispersión exclusivamente abióticos (Fig. 1). La relación entre el sistema reproductivo y el síndrome de dispersión de semillas también fue significativa (Fisher $p=$ 0,0151): las especies xenógamas mostraron síndromes bióticos y abióticos mientras que las autógamas mostraron síndromes exclusivamente abióticos (Fig. 1).

Variabilidad de la eficiencia reproductiva natural entre formas de vida, sistemas reproductivos $y$ síndromes de dispersión de semillas

La relación fruto/flor y la fecundidad relativa fueron mayores en las hierbas que en los árboles, en las plantas autógamas que en las xenógamas 


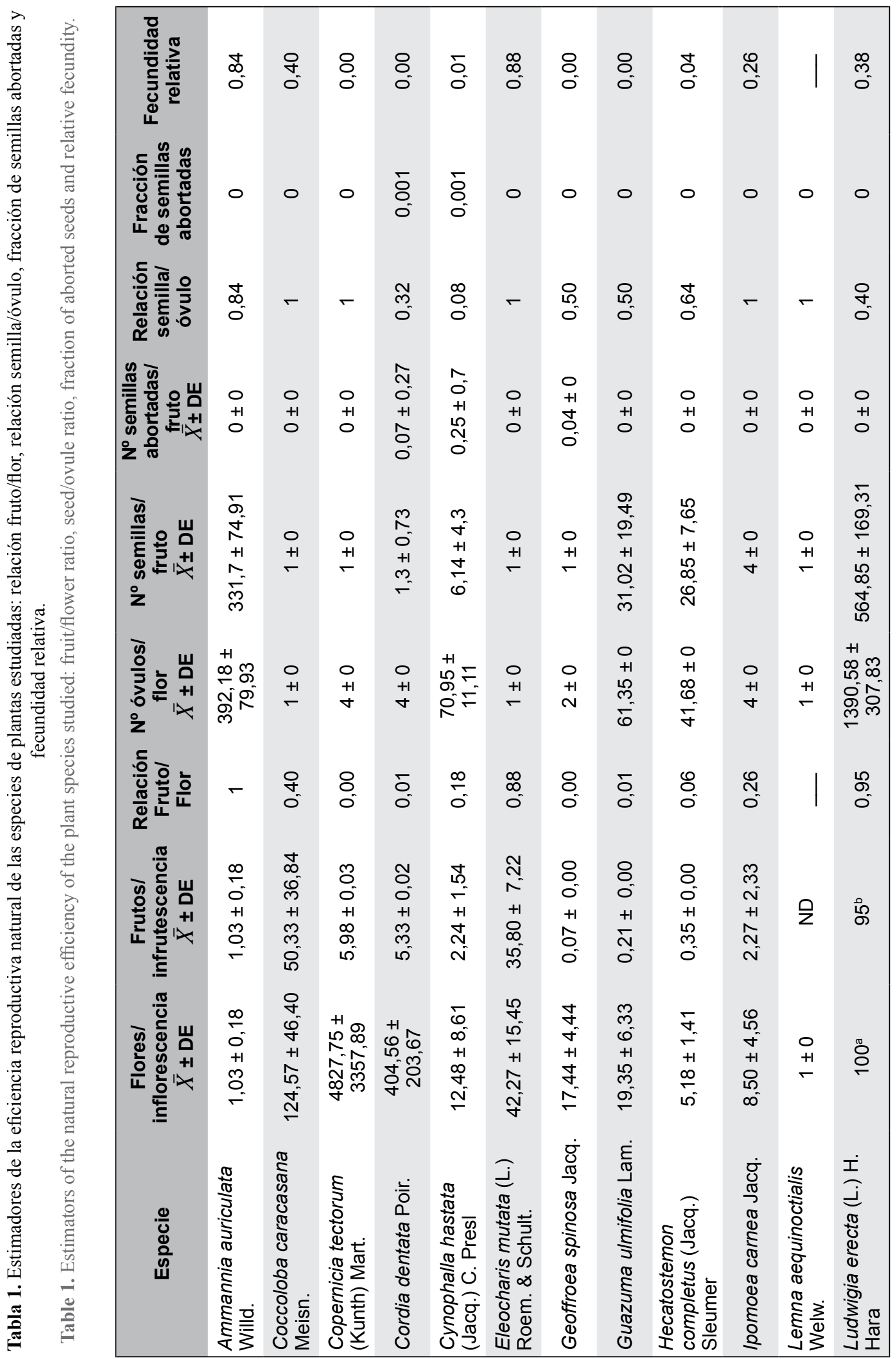




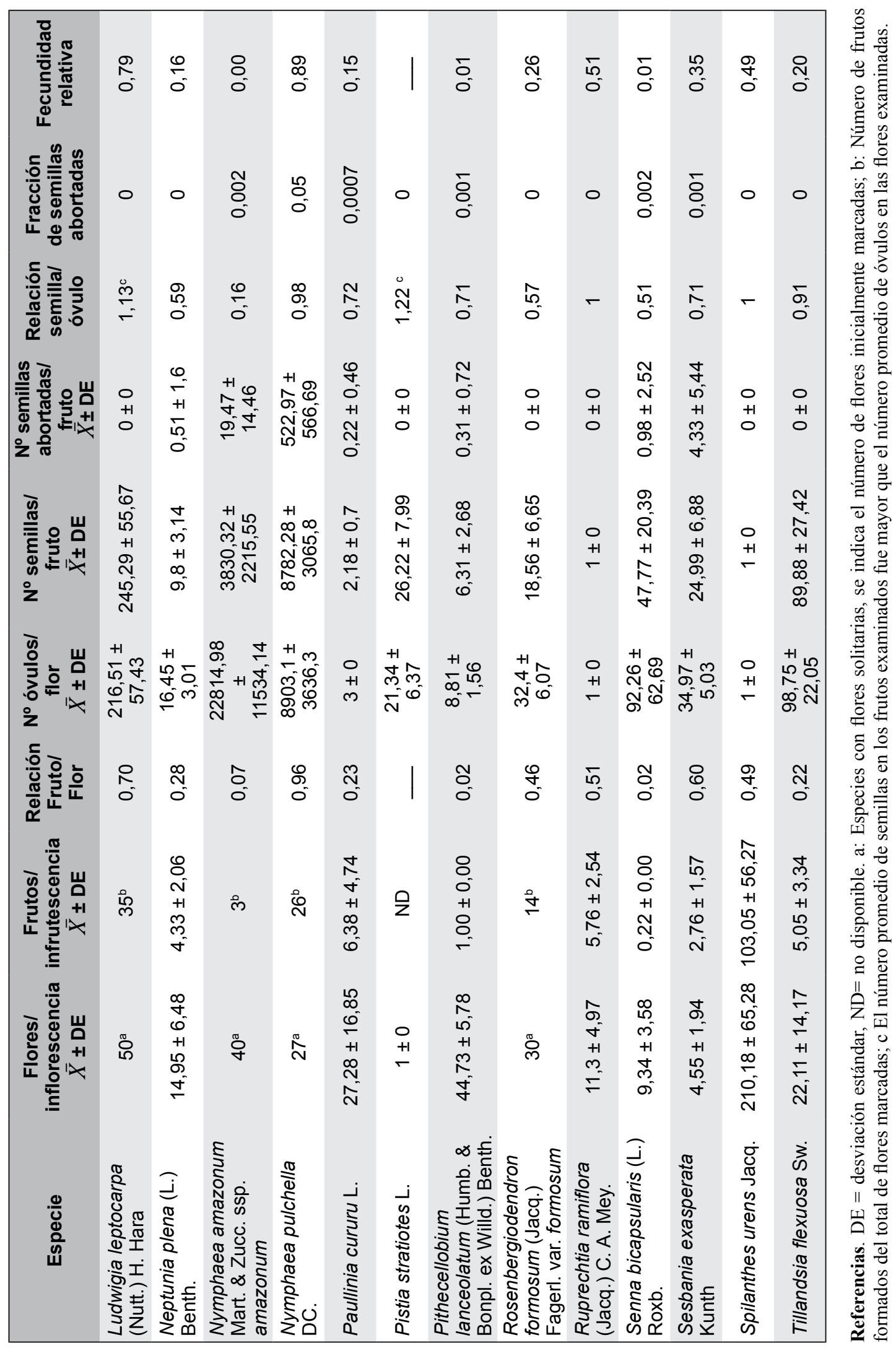


$\mathrm{y}$ en las especies hidrocoras y/o anemocoras que en las zoocoras. La relación semilla/ óvulo fue mayor en las especies hidrocoras y/o anemocoras que en las zoocoras (Tabla 2).
Relación entre la eficiencia reproductiva y el número y costo de las estructuras reproductivas

La relación fruto/flor estuvo negativamente relacionada con el número de flores por

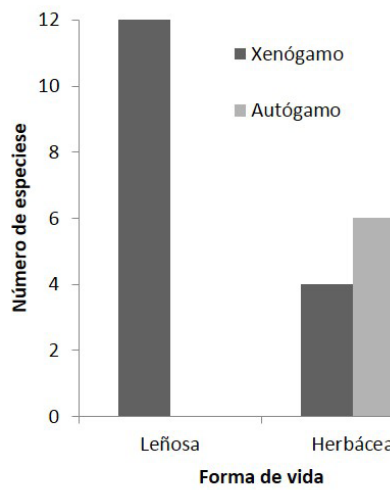

A

B

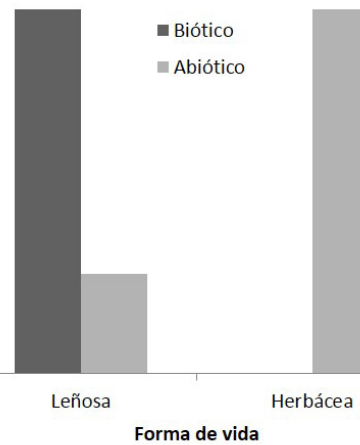

C

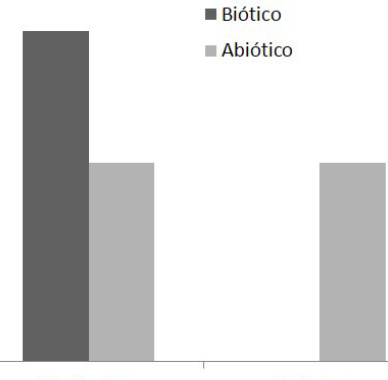

Xenógamo

Sistema reproductivo

Fig. 1. Relación de la forma de vida de las especies con el sistema reproductivo (xenógamo, autógamo) (A) y el tipo de síndrome de dispersión de semillas (biótico, abiótico); (B) así como entre el sistema reproductivo y el tipo de síndrome de dispersión de semillas $(\mathrm{C})$.

Fig. 1. Relationship of the life form of the species to the reproductive system (xenogamous, autogamous) (A) and the type of seed dispersal syndrome (biotic, abiotic); (B) as well as between the reproductive system and the type of seed dispersal syndrome $(\mathrm{C})$.

Tabla 2. Comparación de los estimadores de la eficiencia reproductiva natural entre formas de vida, sistemas reproductivos y síndromes de dispersión de semillas. Para cada análisis se muestra la media $(\bar{X})$, desviación estándar (DE) y el número de especies en cada categoría (n), el valor del estadístico ( $\mathrm{t}$ ) y la probabilidad (p) asociada con la hipótesis nula de que las medias son iguales.

Table 2. Comparison of natural reproductive efficiency estimators between life forms, reproductive systems and seed dispersal syndromes. For each analysis the mean $(\bar{X})$, standard deviation (SD) and the number of species in each category (n),

the statistical value $(\mathrm{t})$ and the probability $(\mathrm{p})$ associated with the null hypothesis that the means are equal are shown.

\begin{tabular}{|c|c|c|c|c|c|c|c|c|c|}
\hline & \multicolumn{3}{|c|}{ Relación fruto/flor } & \multicolumn{3}{|c|}{ Relación semilla/óvulo } & \multicolumn{3}{|c|}{ Fecundidad relativa } \\
\hline & $\bar{X} \pm \mathrm{DE}$ & $\mathbf{n}$ & & $\bar{X} \pm \mathrm{DE}$ & $\mathbf{n}$ & & $\bar{X} \pm \mathrm{DE}$ & $\mathbf{n}$ & \\
\hline \multicolumn{10}{|l|}{ Forma de vida } \\
\hline Leñosa & $\begin{array}{c}0,20 \pm \\
0,24\end{array}$ & 14 & $t=-3,02$ & $\begin{array}{c}0,65 \pm \\
0,20\end{array}$ & 14 & $t=-1,33$ & $0,14 \pm 0,17$ & 14 & $t=-3,20$ \\
\hline Herbácea & $\begin{array}{c}0,57 \pm \\
0,39\end{array}$ & 9 & $\begin{array}{c}(p= \\
0,0064)\end{array}$ & $\begin{array}{c}0,80 \pm \\
0,29\end{array}$ & 11 & $(p=0,1954)$ & $0,51 \pm 0,34$ & 9 & $\begin{array}{c}(p= \\
0,0042)\end{array}$ \\
\hline \multicolumn{10}{|l|}{ Sistema reproductivo } \\
\hline Xenogamia & $\begin{array}{c}0,17 \pm \\
0,24\end{array}$ & 14 & $t=5,18$ & $\begin{array}{c}0,63 \pm \\
0,31\end{array}$ & 14 & $t=1,61$ & $0,15 \pm 0,25$ & 14 & $t=3,79$ \\
\hline Autogamia & $\begin{array}{c}0,90 \pm \\
0,13\end{array}$ & 4 & $\begin{array}{c}(p< \\
0,0000)\end{array}$ & $\begin{array}{c}0,87 \pm \\
0,23\end{array}$ & 6 & $(p=0,1239)$ & $0,72 \pm 0,23$ & 4 & $\begin{array}{c}(p= \\
0,0015)\end{array}$ \\
\hline \multicolumn{10}{|c|}{ Síndrome de dispersión de semillas } \\
\hline $\begin{array}{l}\text { Biótico (ornitocorica y } \\
\text { mamalocoria) }\end{array}$ & $\begin{array}{c}0,12 \pm \\
0,17\end{array}$ & 11 & $t=-3,86$ & $\begin{array}{c}0,59 \pm \\
0,26\end{array}$ & 11 & $t=-2,23$ & $0,08 \pm 0,13$ & 11 & $t=-4,07$ \\
\hline $\begin{array}{l}\text { Abiótico (anemocoria e } \\
\text { hidrocoria) }\end{array}$ & $\begin{array}{c}0,55 \pm \\
0,35\end{array}$ & 12 & $\begin{array}{c}(p= \\
0,0008)\end{array}$ & $\begin{array}{c}0,82 \pm \\
0,26\end{array}$ & 14 & $(p=0,0352)$ & $0,48 \pm 0,30$ & 12 & $\begin{array}{c}(p= \\
0,0005)\end{array}$ \\
\hline
\end{tabular}

Referencia. Valores en negrita indican que la diferencia entre las medias es estadísticamente significativa. 
inflorescencia, con el peso del fruto y de la semilla; la relación semilla/óvulo estuvo negativamente relacionada con el número de óvulos por flor y con el peso del fruto; la fecundidad relativa estuvo negativamente relacionada con el peso del fruto y de la semilla (Tabla 3).

\section{Variabilidad de la biomasa asignada a frutos} $y$ semillas y de la relación pericarpio/semilla entre formas de vida, sistemas reproductivos y síndromes de dispersión de semillas

Los valores de peso seco de frutos y semillas estuvieron positivamente relacionados $(\mathrm{Rs}=$ $0,63, p=0,0036)$. El peso de las semillas fue mayor en las especies leñosas que en las herbáceas, en las xenógamas que en las autógamas, y en las zoocoras que en las hidrocoras y/o anemocoras (Tabla 4). La inversión de biomasa en pericarpio (relación pericarpio/semilla) fue mayor en las especies xenógamas que en las autógamas (Tabla 4).

Tabla 3. Relación entre las variables asociadas con el número y costo de las estructuras reproductivas (número de

flores por inflorescencia, número de óvulos por flor, peso fruto, peso semilla y relación pericarpio/semilla) y los estimadores de la eficiencia reproductiva natural (relación fruto/flor, relación semilla/óvulo y fecundidad relativa). En cada caso se señala el coeficiente de correlación de Spearman (Rs) y la probabilidad (p) asociada con la hipótesis nula de ausencia de correlación entre las variables.

Table 3. Relationship between the variables associated with the number and cost of reproductive structures (number of flowers per inflorescence, number of ovules per flower, fruit weight, seed weight and pericarp/seed ratio) and the estimators of natural reproductive efficiency (fruit/flower ratio, seed/ovule ratio and relative fecundity). In each case, the Spearman correlation coefficient (Rs) and the probability (p) associated with the null hypothesis of no correlation between the variables are indicated.

\begin{tabular}{|c|c|c|c|c|c|c|}
\hline & Relac & ruto/flor & Relac & Ila/óvulo & Fecu & elativa \\
\hline & Rs & $(p)$ & Rs & $(p)$ & Rs & $(p)$ \\
\hline $\begin{array}{l}\text { Número de flores por } \\
\text { inflorescencia }\end{array}$ & $-0,51$ & $(0,01)$ & 0,22 & $(0,29)$ & $-0,29$ & $(0,17)$ \\
\hline Número de óvulos por flor & 0,23 & $(0,28)$ & $-0,50$ & $(0,00)$ & 0,01 & $(0,94)$ \\
\hline Peso fruto (mg) & $-0,55$ & $(0,01)$ & $-0,58$ & $(0,00)$ & $-0,58$ & $(0,00)$ \\
\hline Peso semilla (mg) & $-0,72$ & $(0,00)$ & $-0,18$ & $(0,43)$ & $-0,65$ & $(0,00)$ \\
\hline Relación percarpio/semilla & $-0,23$ & $(0,98)$ & $-0,24$ & $(0,30)$ & $-0,09$ & $(0,71)$ \\
\hline
\end{tabular}

Referencia. Valores en negrita indican que la correlación es estadísticamente significativa.

Tabla 4. Comparación de los valores de biomasa asignada a frutos y semillas y de la relación pericarpio/semilla de las especies a nivel de formas de vida, sistemas reproductivos y síndromes de dispersión de semillas. Para cada análisis se muestra la media $(\bar{X})$, desviación estándar (DE) y el número de especies en cada categoría (n), el valor del estadístico (t o Welch) y la probabilidad (p) asociada con la hipótesis nula de que las medias son iguales.

Table 4. Comparison of the biomass values assigned to fruits and seeds and the pericarp/seed ratio of species at the

level of life forms, reproductive systems and seed dispersal syndromes. For each analysis the mean $(\bar{X})$, standard deviation (SD) and the number of species in each category (n), the statistical value ( $\mathrm{t}$ or Welch) and the probability (p) associated with the null hypothesis that the means are equal are shown.

\begin{tabular}{|c|c|c|c|c|c|c|c|c|c|}
\hline & \multicolumn{3}{|c|}{ Peso seco fruto $(\mathrm{mg})$} & \multicolumn{3}{|c|}{ Peso seco semillas (mg) } & \multicolumn{3}{|c|}{$\begin{array}{c}\text { Relación pericarpio/ } \\
\text { semilla }\end{array}$} \\
\hline & $\bar{X} \pm \mathrm{DE}$ & $\mathbf{n}$ & & $\bar{X} \pm \mathrm{DE}$ & $\mathbf{n}$ & & $\bar{X} \pm \mathrm{DE}$ & $\mathbf{n}$ & \\
\hline \multicolumn{10}{|l|}{ Forma de vida } \\
\hline Leñosa & $\begin{array}{c}1351,76 \pm \\
1385,27\end{array}$ & 12 & $t=-1,38$ & $\begin{array}{c}275,61 \pm \\
492,97\end{array}$ & 11 & $\begin{array}{c}\text { Welch }= \\
-2,70\end{array}$ & $\begin{array}{c}2,03 \pm \\
1,21\end{array}$ & 10 & $\begin{array}{c}\text { Welch }= \\
-0,72\end{array}$ \\
\hline Herbácea & $\begin{array}{c}1044,82 \pm \\
2540,10\end{array}$ & 10 & $\begin{array}{c}(p= \\
0,1802)\end{array}$ & $1,07 \pm 2,12$ & 10 & $\begin{array}{c}(p= \\
0,0220)\end{array}$ & $\begin{array}{c}1,83 \pm \\
3,81\end{array}$ & 9 & $\begin{array}{c}(p= \\
0,4830)\end{array}$ \\
\hline
\end{tabular}




\begin{tabular}{|c|c|c|c|c|c|c|c|c|c|}
\hline & \multicolumn{3}{|c|}{ Peso seco fruto $(\mathrm{mg})$} & \multicolumn{3}{|c|}{ Peso seco semillas (mg) } & \multicolumn{3}{|c|}{$\begin{array}{c}\text { Relación pericarpio/ } \\
\text { semilla }\end{array}$} \\
\hline & $\bar{X} \pm \mathrm{DE}$ & $\mathbf{n}$ & & $\bar{X} \pm \mathrm{DE}$ & $\mathbf{n}$ & & $\bar{X} \pm \mathrm{DE}$ & $\mathbf{n}$ & \\
\hline \multicolumn{10}{|l|}{ Sistema reproductivo } \\
\hline Xenogamia & $\begin{array}{c}1383,05 \pm \\
1356,95\end{array}$ & 13 & $t=-0,73$ & $\begin{array}{c}229,18 \pm \\
463,73\end{array}$ & 13 & $\begin{array}{c}\text { Welch }= \\
-2,39\end{array}$ & $\begin{array}{c}2,76 \pm \\
3,12\end{array}$ & 13 & $\begin{array}{c}\text { Welch }= \\
-3,30\end{array}$ \\
\hline Autogamia & $\begin{array}{c}1633,89 \pm \\
3592,37\end{array}$ & 5 & $\begin{array}{c}(p= \\
0,4733)\end{array}$ & $0,44 \pm 0,51$ & 5 & $\begin{array}{c}(p= \\
0,0335)\end{array}$ & $\begin{array}{c}0,39 \pm \\
0,28\end{array}$ & 4 & $\begin{array}{c}(p= \\
0,0051)\end{array}$ \\
\hline \multicolumn{10}{|c|}{ Síndrome de dispersión de semillas } \\
\hline $\begin{array}{l}\text { Biótico (ornitocoria y } \\
\text { mamalocoria) }\end{array}$ & $\begin{array}{c}1457,23 \pm \\
1401,43\end{array}$ & 11 & $t=-1,61$ & $\begin{array}{c}326,07 \pm \\
536,22\end{array}$ & 9 & $\begin{array}{c}\text { Welch }= \\
-2,34\end{array}$ & $\begin{array}{c}2,17 \pm \\
1,20\end{array}$ & 9 & $\begin{array}{c}\text { Welch }= \\
-1,00\end{array}$ \\
\hline $\begin{array}{c}\text { Abiótico (anemocoria e } \\
\text { hidrocoria) }\end{array}$ & $\begin{array}{l}967,25 \pm \\
2423,45\end{array}$ & 11 & $\begin{array}{c}(p= \\
0,1209)\end{array}$ & $\begin{array}{l}8,98 \pm \\
26,49\end{array}$ & 12 & $\begin{array}{c}(p= \\
0,0458)\end{array}$ & $\begin{array}{c}1,72 \pm \\
3,61\end{array}$ & 10 & $\begin{array}{c}(p= \\
0,3321)\end{array}$ \\
\hline
\end{tabular}

Referencia. Valores en negrita indican que la diferencia entre las medias es estadísticamente significativa.

\section{Discusión}

La baja eficiencia reproductiva de las especies de plantas estudiadas puede ser inicialmente explicada por las hipótesis basadas en la aptitud femenina de las plantas, específicamente por el número y costo de las estructuras reproductivas. A medida que aumenta el número de flores por inflorescencia disminuye el número de frutos que pueden ser desarrollados. Lo mismo pasa con la biomasa asignada a frutos y semillas ya que los recursos disponibles para la planta son limitados (Stephenson, 1981; Sutherland, 1986; Ramírez, 1992, 1993; Ramírez et Berry, 1993). La correlación negativa de la relación fruto/flor con el número de flores por inflorescencia, con el peso del fruto y de la semilla apoyan este planteamiento. Así como el hecho de que la mayor relación fruto/flor en especies herbáceas, autógamas $\mathrm{y}$ de dispersión abiótica coincide con los menores valores de biomasa que presentan las semillas de estas especies frente a sus contrapartes leñosas, xenógamas y zoocoras, respectivamente.

La variabilidad de la biomasa asignada a las semillas a nivel de formas de vida y su reflejo en la eficiencia reproductiva coincide con los resultados de Ramírez (1993). Este autor señala que la baja asignación de biomasa a frutos y semillas, así como los altos valores de peso de la flor en comparación con el peso del fruto y el peso de las semillas por fruto, favorece la fecundidad relativa en las hierbas.
En los árboles, en cambio, se producen frutos y semillas de alto valor energético a expensas de elevados niveles de aborto ya que el costo de los frutos impone un límite al número de frutos que pueden ser producidos por inflorescencia. La mayor producción de frutos y semillas por inflorescencia que mostraron las especies herbáceas respecto a las leñosas en este trabajo apoya entonces la noción propuesta por Ramírez (1993) de que el incremento en el costo energético de las estructuras reproductivas promueve mayores niveles de aborto desde formas de vida herbáceas a formas de vida leñosas.

La variabilidad del costo de las estructuras reproductivas a nivel de sistemas reproductivos guarda relación con lo registrado entre formas de vida. El bajo peso que presentaron las semillas de las especies autógamas, al igual que la escasa inversión de biomasa en pericarpio (materna), refleja el bajo costo asociado con las estructuras reproductivas de las especies herbáceas y está relacionado con la mayor producción de frutos y semillas por inflorescencia que muestran las especies autógamas respecto a las xenógamas. La asociación entre la autogamia y el hábito herbáceo ha sido ampliamente señalada (Cruden, 1977; Ramírez et Brito, 1990; Lloyd et Schoen, 1992; Lemus-Jiménez et Ramírez, 2005), igual que la de la autogamia con el bajo peso del pericarpio y de las semillas (Galen et al., 1985; Galen et Weger, 1986; Uma Shaanker et al., 1988; Fenster, 1991; Götzenberger et 
al., 2006; Burd, 2011). Sin embargo, este no es el único factor relacionado con las diferencias registradas en la producción de frutos y semillas por inflorescencia a nivel de sistemas reproductivos. En este sentido, se ha señalado que la seguridad de la autopolinización va acompañada de un incremento en la aptitud femenina (Cruden, 1977; Brunet, 1992). En cambio, las especies hermafroditas xenógamas, más allá de estar limitadas por la disponibilidad de polen exógeno (Burd, 1994; Ashman et al., 2004), presentan niveles elevados de aborto de frutos y además, pueden sufrir los efectos negativos de las reacciones de autoincompatibilidad (Sutherland et Delph, 1984; Sutherland, 1986; Wiens et al., 1987; Uma Shaanker et al., 1988), factores que redundan en baja eficiencia reproductiva.

Las diferencias registradas entre síndromes de dispersión de semillas también coinciden con lo esperado de acuerdo con la hipótesis del costo de las estructuras reproductivas. En este sentido, la baja producción de frutos y semillas por inflorescencia, así como de semillas por óvulo, que presentaron las especies zoocoras, parece reflejar la mayor asignación de biomasa a nivel de semillas que muestran estas especies frente a sus contrapartes de dispersión abiótica las cuales exhibieron alta eficiencia reproductiva. El elevado costo energético de las diásporas zoocoras puede ser explicado por las características morfológicas (tamaño, forma) y bioquímicas (color, olor, composición nutricional de la recompensa) que promueven la atracción y el consumo por parte de los animales dispersores (Ramírez et Berry, 1993; Herrera, 2002). Además, la zoocoria estuvo asociada con las formas de vida leñosas y con la xenogamia, atributos que también están relacionados con una alta asignación de biomasa a nivel de semillas y con una baja eficiencia en la producción de frutos y de semillas por inflorescencia.

En cuanto a la eficiencia en la producción de semillas por óvulo, la asociación negativa entre la relación semilla/óvulo y el peso del fruto sugiere que la producción de frutos de alto costo energético, además de disminuir la proporción de frutos producidos por inflorescencia, afecta la proporción de semillas producidas por fruto. Este puede ser el caso de frutos con una elevada asignación de biomasa en pericarpio y/o a nivel de semillas. La cantidad de semillas que puede ser desarrollada en estos frutos se reduce dado que los recursos son limitados. La progenie es seleccionada por la competencia entre óvulos, antes y/o después de la fertilización lo que deriva en la interrupción del desarrollo del óvulo, e.g. de la formación del saco embrionario, o en el aborto del óvulo fecundado, respectivamente (Mogensen, 1975; Lloyd, 1980; Stephenson, 1981; Bawa et Weeb, 1984; Galen et Weger, 1986; Nakamura, 1988; Uma Shaanker et al., 1988; Fenster, 1991; Rocha et Stephenson, 1991; Ramírez, 1992, 1993). Aunque en este último caso se ha sugerido que al igual que acontece con los frutos (Stephenson, 1981), además de la competencia, existe aborto selectivo por parte de la planta madre (Korbecka et al., 2002). Incluso se ha propuesto que más allá de maximizar la calidad genética de la progenie, el aborto está dirigido a homogeneizar la distribución de recursos entre los óvulos fecundados que inician su desarrollo (Sakai, 2007).

Otra hipótesis propuesta para explicar la diferencia entre el número de semillas por fruto y el número de óvulos por flor establece que al igual que existe una sobreproducción de flores que funcionan como compensación ante la disponibilidad de polinizadores, existe un exceso de óvulos en las flores que permite compensar las variaciones en la disponibilidad de tubos polínicos (Lloyd, 1980; Stephenson, 1981; Bawa et Weeb, 1984; Ramírez, 1992; Burd, 1994, 1995, 2009; Porcher et Lande, 2005). La polinización suplementaria en varias especies ha puesto a prueba esta hipótesis y no la ha rechazado, ya que el tratamiento incrementa el número de semillas producidas (Burd, 1994; Ashman et al., 2004). Sin embargo, dadas las características de la comunidad de plantas estudiada, la producción de semillas no parece estar considerablemente limitada por polen. Las visitas florales en la mayoría de las especies fueron frecuentes y los polinizadores mostraron altos niveles de 
especificidad de carga y transporte de polen (Barrios, 2017). La limitación de recursos, por lo tanto, parece una hipótesis más verosímil para explicar, tanto la proporción de flores que no se transforman en frutos, como de óvulos que no se transforman en semillas (Lloyd, 1980; Stephenson, 1981; Bawa et Weeb, 1984; Harder et al., 2008). En el caso de especies con numerosos óvulos por flor, los recursos pueden volverse limitantes de forma impredecible y causar altos niveles de aborto de óvulos (Lloyd, 1980; Bawa et Weeb, 1984; Uma Shaanker et al., 1988). Cuando el número de óvulos es bajo, en cambio, el fruto entero puede ser la unidad de selección, de tal modo que abortaría si los recursos son limitados (Uma Shaanker et al., 1988; Ramírez, 1992; Ramírez et Berry, 1993). De aquí, la asociación negativa entre la relación semilla/óvulo y el número de óvulos por flor en las especies estudiadas.

El aborto de semillas, por ser escaso o nulo, puede ser considerado un evento irrelevante durante la reproducción de las especies estudiadas. Así lo describe Ramírez (1992) quien también registró una baja fracción de semillas abortadas $(0-0,2)$ en la mayoría de las especies incluidas en su investigación. Dados estos niveles tan exiguos, el autor propone que factores extrínsecos, energéticamente no previstos durante la fertilización de los óvulos y el desarrollo de las semillas, son los responsables del aborto de estas estructuras. No obstante, en un trabajo más reciente, Ramírez et Herrera (2017) señalaron que el aborto de semillas está correlacionado negativamente con el contenido de nitrógeno en las hojas, lo que sugiere a los nutrientes en la planta como factores asociados con el aborto de semillas. Por otra parte, también se ha asociado al aborto de semillas con la expresión de alelos deletéreos y subletales en estados avanzados de desarrollo del embrión, aunque es más probable que éstos se manifiesten en etapas más tempranas como aborto de óvulos fecundados (Nakamura et Stanton, 1987; Wiens et al., 1987). De cualquier manera, comparado con la proporción de flores no polinizadas y/o de frutos abortados, y en segundo lugar, con la proporción de óvulos no fertilizados y/o que abortan una vez fecundados, el aborto en la fase de semillas parece ser de bajo valor selectivo en la producción de semillas sanas por inflorescencia.

Finalmente, los resultados de este trabajo indican que la eficiencia en la producción de frutos y semillas está fuertemente influenciada por el costo de estas estructuras. Las especies herbáceas, autógamas y/o con dispersión abiótica, dado el bajo costo de sus semillas, se reproducen más eficientemente en concordancia con una estrategia $r$ (Taylor, 1990). Las especies arbóreas, xenógamas y/o zoocoras, en cambio, invierten mayor cantidad de recursos en las estructuras reproductivas pero presentan elevados niveles de aborto de frutos y en el caso de las especies zoocoras también una baja relación semilla/óvulo. Aunque esto posiblemente se compensa con la mayor variabilidad genética y longevidad de las semillas así como con la mayor especificidad que puede llegar a tener la dispersión por vertebrados (Herrera, 2002), estrategia k (Taylor, 1990). Ambos tipos de estrategias (r y k) son importantes en la comunidad estudiada. La elevada eficiencia reproductiva de las especies herbáceas, autógamas y/o con dispersión abiótica, siendo estas mayormente plantas de humedales ( $c f$. Velásquez, 1994), representa una ventaja frente al régimen hídrico. Las plantas pueden dejar suficiente cantidad de progenie tras cada ciclo de sequía/inundación. En tanto que la mayor inversión de recursos en las estructuras reproductivas de especies arbóreas, xenógamas y/o zoocoras, la mayoría de sustratos secos o inundados por corto tiempo, puede garantizarle mayor probabilidad de sobrevivencia a las semillas de estas especies en hábitats boscosos que son más competitivos (Taylor, 1990). Los diferentes patrones de eficiencia reproductiva encontrados en este trabajo reflejan entonces las estrategias que prevalecen entre los dos hábitats extremos de un bosque estacionalmente inundable.

\section{Agradecimientos}

Los autores agradecen a N. H. Barrios, J. O. Zambrano, J. A. Soto y al Sr. Fabricio (baquiano) por su colaboración en las actividades de campo; a J. O. Zambrano y J. R. Grande por la identificación de las especies de plantas; y a dos 
revisores anónimos por sus valiosos comentarios y sugerencias para mejorar el manuscrito. Este trabajo es parte de la tesis doctoral de la primera autora y fue realizado gracias al incentivo institucional de la Misión Ciencia en Venezuela (año 2007, contrato $n^{\circ}$ 200900537) al Laboratorio de Biología Reproductiva de Angiospermas (Instituto de Biología Experimental, Universidad Central de Venezuela).

\section{Bibliografía}

APG IV. (2016). An update of the Angiosperm Phylogeny Group classification for the orders and families of flowering plants: APG IV. Botanical Journal of the Linnean Society 181: 1-20. https://doi.org/10.1111/boj.12385

ASHMAN, T. L., T. M. KNIGHT, J. STEETS, P. AMARASEKARE, M. BURD, R. CAMPBELL, M. R. DUDASH, M. O. JOHNSTON, S. J. MAZER, R. J. MITCHELL, M. T. MORGAN \& W. G. WILSON. (2004). Pollen limitation of plant reproduction: Ecological and evolutionary causes and consequences. Ecology 85: 2408-2421. https:// doi: 10.1890/03-8024.

BARRIOS, Y. (2017). Caracterización reproductiva de las angiospermas de un humedal de la costa oriental del lago de Maracaibo, estado Zulia, Venezuela. Tesis doctoral, Universidad Central de Venezuela, Venezuela. $161 \mathrm{pp}$.

BARRIOS, Y. \& N. RAMÍREZ. (2008). Depresión por exogamia y biología reproductiva de Nymphaea ampla (Salisb.) DC. (Nymphaeaceae). Acta Botánica Venezuelica 31: 539-556.

BAWA, K. S. (1974). Breeding systems of tree species of a lowland tropical community. Evolution 28: 85-92. https://doi.org/10.1111/j.1558-5646.1974.tb00729.x

BAWA, K. S. \& C. J. WEEB. (1984). Flower, fruit and seed abortion in tropical forest trees: Implications for the evolution of paternal and maternal reproductive patterns. American Journal of Botany 71: 736-751. https://doi.org/10.1002/j.1537-2197.1984.tb14181.x

BRUNET, J. (1992). Sex allocation in hermaphroditic plants. Trends in Ecology \& Evolution 7: 79-84. https://doi.org/10.1016/0169-5347(92)90245-7

BULLOCK, S. H. (1985). Breeding systems in the flora of a tropical deciduous forest in Mexico. Biotropica 17: 287-301. https://doi.org/10.2307/2388591
BURD, M. (1994). Bateman's principle and plants reproduction. The role of pollen limitation in fruit and seed set. The Botanical Review 60: 84-139. https://doi.org/10.1007/BF02856594

BURD, M. (1995). Ovule packaging in stochastic pollination and fertilization environments. Evolution 48: 55-68. https://doi.org/10.2307/2410296

BURD, M. (2004). Offspring quality in relation to excess flowers in Pultenaea gunnii (Fabaceae). Evolution 58: 2371-2376. https://doi.org/10.1111/j.0014-3820.2004.tb01610.x

BURD, M. (2009). Ovule number per flower in a world of unpredictable pollination. American Journal of Botany 96: 1159-1167. https://doi.org/10.3732/ajb.0800183

BURD, M. (2011). Are relationships between pollenovule ratio and pollen and seed size explained by sex allocation? Evolution 65: 3002-3005. https://doi.org/10.1111/j.1558-5646.2011.01338.x

CHARLESWORTH, D. \& B. CHARLESWORTH. (1987). Inbreeding depression and its evolutionary consequences. Annual Review of Ecology, Evolution, and Systematics 18: 237-268.

https://doi.org/10.1146/annurev.es.18.110187.001321

CRUDEN, R. (1977). Pollen-ovule ratios: A conservative indicator of breeding systems in flowering plants. Evolution 31: 32-46. https://doi.org/10.2307/2407542 https://doi.org/10.1111/j.1558-5646.1977.tb00979.x

FENSTER, C. (1991). Effect of male pollen donor and female seed parent on allocation of resources to developing seeds and fruit in Chamaecrista fasciculata (Leguminosae). American Journal of Botany 78: 13-23. https://doi.org/10.1002/j.1537-2197.1991.tb12567.x

GALEN, C., R. C. PLOWRIGHT \& J. D. THOMSON. (1985). Floral biology and regulation of seed set and seed size in the lily Clintonia borealis. American Journal of Botany 72: 1544-1552. https://doi.org/10.1002/j.1537-2197.1985.tb08418.x

GALEN, C. \& H. WEGER. (1986). Re-evaluating the significance of correlations between seed number and size: evidence from a natural population of the lily, Clintonia borealis. American Journal of Botany 73: 346-352. https://doi.org/10.1002/j.1537-2197.1986.tb12047.x

GÖTZENBERGER, L., W. DURKA, I. KÜHN \& S. KLOTZ. (2006). The relationship of pollenovule ratio and seed size: a comparative test of a sex allocation hypothesis. Evolutionary Ecology Research 8: 1101-1116.

GRAHAM, S. (2003). Biogeographic Patterns of Antillean Lythraceae. Systematic Botany 28: 410-420. 
HAMMER, Ø., D. A. T. HARPER \& P. D. RYAN. (2001). PAST: Paleontological statistics software package for education and data analysis. Paleontología Electrónica 4(1). 9 pp. http://palaeo-electronica. org/2001_1/past/issue1_01.htm

HARDER, L. D., S.A. RICHARDS \& M. B. ROUTLEY. (2008). Effects of reproductive compensation, gamete discounting and reproductive assurance on mating-systems diversity in hermaphrodites. Evolution 62: 157-172. https://doi.org/10.1111/j.1558-5646.2007.00272.x

HERRERA, C. M. (2002). Seed dispersal by vertebrates. En HERRERA C. M. \& O. PELLMYR (eds.), PlantAnimal interactions. An evolutionary approach, pp. 185-208. Oxford: Blackwell Science.

HUSBAND, B. C. \& D. W. SCHEMSKE. (1996). Evolution of the magnitude and timing of inbreeding depression in plants. Evolution 50: 54-70. https://doi.org/10.1111/j.1558-5646.1996.tb04472.x

KORBECKA, G., P. G. L. KLINKHAMER \& K. VRIELING. (2002). Selective embryo abortion hypothesis revisited - a molecular approach. Plant Biology 4: 298-310. https://doi.org/10.1055/s-2002-32331

LANDOLT, E. (1986). Biosystematics investigations in the family of duckweeds (Lemnaceae). The family of Lemnaceae, a monographic study. Vol I. Veröffentlichungen des Geobotanischen Institute der ETH, Stiftung Rübel, Zürich, Switzerland. 566 pp.

LEMUS-JIMÉNEZ, L. \& N. RAMÍREZ. (2005). Sistemas reproductivos de las plantas en tres hábitats de la planicie costera de Paraguaná. Revista de Biología Tropical 53: 415-430. https://doi.org/10.15517/rbt.v53i3-4.14610

LLOYD, D. (1980). Sexual strategies in plants II. An hypothesis of serial adjustment of maternal investment during one reproductive session. New Phytologist 86: 69-80. https://doi.org/10.1111/j.1469-8137.1980.tb00780.x

LLOYD, D. \& D. SCHOEN. (1992). Self-and crossfertilization in plants. I. Functional dimensions. International Journal of Plant Sciences 153: 358369. https://doi.org/10.1086/297040

MOGENSEN, H. L. (1975). Ovule abortion in Quercus (Fagaceae). American Journal of Botany 62: 160-165. https://doi.org/10.1002/j.1537-2197.1975.tb14047.x

NAKAMURA, R. R. (1988). Seed abortion and seed size variation within fruits of Phaseolus vulgaris: pollen donor and resource limitation effects. American Journal of Botany 75: 1003-1010. https://doi.org/10.1002/j.1537-2197.1988.tb08807.x
NAKAMURA, R. R. \& M. L. STANTON. (1987). Cryptic seed abortion and the estimation of ovule fertilization. Canadian Journal of Botany 65: 2463 -2465. https://doi.org/10.1139/b87-334

OPLER, P. A., H. G. BAKER \& G. W. FRANKIE. (1975). Reproductive biology of some Costa Rican Cordia species (Boraginaceae). Biotropica 7: 234-247. https://doi.org/10.2307/2989736

OZIEGBE, M. \& J. O. FALUYI. (2012). Reproductive biology of Ludwigia leptocarpa and L. adscendens subsp. diffusa in Ile Ife, Nigeria. Turkish Journal of Botany 36: 167-173. https://doi:10.3906/bot-1101-21

PEEL, M. C., B. L. FINLAYSON \& T. A. MCMAHON. (2007). Updated world map of the Köppen-Geiger climate classification. Hydrology and Earth System Sciences 11: 1633-1644. https://doi.org/10.5194/hess-11-1633-2007

PORCHER, E. \& R. LANDE. (2005). Reproductive compensation in the evolution of plant mating systems. New Phytologist 166: 673-684. https://doi.org/10.1111/j.1469-8137.2005.01363.x

RAMÍREZ, N. (1992). Las características de las estructuras reproductivas, niveles de aborto y semillas producidas. Acta Científica Venezolana 43: 167-177.

RAMÍREZ, N. (1993). Producción y costo de frutos y semillas entre formas de vida. Biotropica 25: 46-60. https://doi.org/10.2307/2388978

RAMÍREZ, N. \& P. BERRY. (1993). Producción y costo de frutos y semillas relacionados a los tipos morfológicos de frutos, unidad de dispersión y síndrome de dispersión. Ecotropicos 6: 43-61.

RAMÍREZ, N. \& Y. BRITO. (1990). Reproductive biology of a tropical palm swamp community in the Venezuelan Llanos. American Journal of Botany 77: 1260-1271. https://doi.org/10.1002/j.1537-2197.1990.tb11378.x

RAMÍREZ, N. \& A. HERRERA. (2017). Reproductive efficiency and photosynthetic pathway in seed plants. Perspectives in Plant Ecology Evolution and Systematics 24: 48-60. https://doi: 10.1016/j. ppees.2016.12.004

https://doi.org/10.1016/j.ppees.2016.12.004

ROCHA, O. J. \& A. G. STEPHENSON. (1991). Effects of nonrandom seed abortion on progeny performance in Phaseolus coccineus L. Evolution 45: 1198-1208. https://doi.org/10.1111/j.1558-5646.1991.tb04386.x https://doi.org/10.2307/2409727

RUIZ-ZAPATA, T. \& M. T. K. ARROYO. (1978). Plant reproductive ecology of a secondary deciduous forest in Venezuela. Biotropica 10: 221-230. https://doi.org/10.2307/2387907 
SAKAI, S. (2007). A new hypothesis for the evolution of overproduction of ovules: an advantage of selective abortion for females not associated with variation in genetic quality of the resulting seeds. Evolution 61: 984-993. https://doi:10.1111/j.15585646.2007.00083.x https://doi.org/10.1111/j.1558-5646.2007.00083.x

SOKAL, R. R. \& J. ROHLF. (1969). Biometry. W. H. Freeman \& Company, San Francisco, California. $776 \mathrm{pp}$.

STEPHENSON, A. (1981). Flower and fruit abortion: Proximate causes and ultimate functions. Annual Review of Ecology, Evolution, and Systematics 12: 252-279. https://doi.org/10.1146/annurev. es.12.110181.001345

SUTHERLAND, S. (1986). Patterns of fruit-set: What controls fruit-flower ratios in plants? Evolution 40: 117-128. https://doi.org/10.1111/j.1558-5646.1986.tb05723.x

SUTHERLAND, S. \& L. DELPH. (1984). On the importance of male fitness in plants: Patterns of fruit set. Ecology 65: 1092-1104. https://doi.org/10.2307/1938317
TAYLOR, D. R. (1990). On the relationship between $r / K$ selection and environmental carrying capacity: A new habitat templet for plant life history strategies. Oikos 58: 239-250. https://doi.org/10.2307/3545432

UMA SHAANKER, R., K. N. GANESHAIAH \& K. S. BAWA. (1988). Parent-Offspring Conflict, Sibling Rivalry, and Brood Size Patterns in Plants. Annual Review of Ecology, Evolution, and Systematics 19: 177-205. https://doi.org/10.1146/annurev.es.19.110188.001141

VAN DER PIJL, L. (1972). Principles of dispersal in higher plants. Second edition. Springer-Verlag. Berlin. $161 \mathrm{pp}$. https://doi.org/10.1007/978-3-642-96108-3

VELÁSQUEZ, J. (1994). Plantas acuáticas vasculares de Venezuela. Universidad Central de Venezuela. Consejo de Desarrollo Científico y Humanístico. Caracas, Venezuela. 992 pp.

WIENS, D., C. L. CALVIN, C. A. WILSON, C. I. DAVERN, D. FRANK \& S. R. SEAVEY. (1987). Reproductive success, spontaneous embryo abortion and genetic load in flowering plants. Oecologia 71: 501-509. https://doi.org/10.1007/BF00379288 
BONPLANDIA 29(1). 2020

Anexo 1. Especies de plantas estudiadas. En cada caso se señala la familia, forma de vida, sistema sexual y síndrome de dispersión de semillas.

Annex 1. Species of plants studied. In each case, the family, life form, sexual system and seed dispersal syndrome are indicated.

\begin{tabular}{|c|c|c|c|}
\hline $\begin{array}{l}\text { Familia } \\
\text { Especie }\end{array}$ & $\begin{array}{l}\text { Forma de } \\
\text { vida }\end{array}$ & Sistema sexual & $\begin{array}{l}\text { Síndrome de dispersión } \\
\text { de semillas }\end{array}$ \\
\hline \multicolumn{4}{|l|}{ Araceae } \\
\hline Lemna aequinoctialis Welw. & Hierba & Monoico & Hidrocoria \\
\hline Pistia stratiotes L. & Hierba & Monoico & Hidrocoria \\
\hline \multicolumn{4}{|l|}{ Arecaceae } \\
\hline Copernicia tectorum (Kunth) Mart. & Árbol & Hermafrodita & Mamalocoria \\
\hline \multicolumn{4}{|l|}{ Asteraceae } \\
\hline Spilanthes urens Jacq. & Hierba & Hermafrodita & Anemocoria \\
\hline \multicolumn{4}{|l|}{ Boraginaceae } \\
\hline Cordia dentata Poir. & Árbol & Hermafrodita & Ornitocoria \\
\hline \multicolumn{4}{|l|}{ Bromeliaceae } \\
\hline Tillandsia flexuosa Sw. & Epífita & Hermafrodita & Anemocoria \\
\hline \multicolumn{4}{|l|}{ Capparaceae } \\
\hline Cynophalla hastata (Jacq.) J. Presl & Árbol & Hermafrodita & Ornitocoria \\
\hline \multicolumn{4}{|l|}{ Convolvulaceae } \\
\hline Ipomoea carnea Jacq. & Arbusto & Hermafrodita & Anemocoria \\
\hline \multicolumn{4}{|l|}{ Cyperaceae } \\
\hline Eleocharis mutata (L.) Roem. \& Schult. & Hierba & Hermafrodita & Anemocoria $^{\mathrm{a}}$ - Hidrocoria \\
\hline \multicolumn{4}{|l|}{ Fabaceae } \\
\hline Geoffroea spinosa Jacq. & Árbol & Hermafrodita & Mamalocoria ${ }^{a}$ - Hidrocoria \\
\hline Neptunia plena (L.) Benth. & Sufrútice & Hermafrodita $^{b}$ & Hidrocoria \\
\hline $\begin{array}{l}\text { Pithecellobium lanceolatum (Humb. \& Bonpl. ex } \\
\text { Willd.) Benth. }\end{array}$ & Árbol & Hermafrodita & Ornitocoria \\
\hline Senna bicapsularis (L.) Roxb. & Arbusto & Hermafrodita & Mamalocoria \\
\hline Sesbania exasperata Kunth & Arbusto & Hermafrodita & Hidrocoria \\
\hline \multicolumn{4}{|l|}{ Flacourtiaceae } \\
\hline Hecatostemon completus (Jacq.) Sleumer & Arbusto & Hermafrodita & Ornitocoria \\
\hline \multicolumn{4}{|l|}{ Lythraceae } \\
\hline Ammannia auriculata Willd. & Hierba & Hermafrodita & Hidrocoria \\
\hline \multicolumn{4}{|l|}{ Malvaceae } \\
\hline Guazuma ulmifolia Lam. & Árbol & Hermafrodita & Mamalocoria ${ }^{a}$ - Hidrocoria \\
\hline \multicolumn{4}{|l|}{ Nymphaeaceae } \\
\hline Nymphaea amazonum Mart. \& Zucc. ssp. amazonum & Hierba & Hermafrodita & Hidrocoria \\
\hline Nymphaea pulchella DC. & Hierba & Hermafrodita & Hidrocoria \\
\hline \multicolumn{4}{|l|}{ Onagraceae } \\
\hline Ludwigia erecta (L.) H. Hara & Sufrútice & Hermafrodita & Hidrocoria \\
\hline Ludwigia leptocarpa (Nutt.) H. Hara & Sufrútice & Hermafrodita & Hidrocoria \\
\hline \multicolumn{4}{|l|}{ Polygonaceae } \\
\hline Coccoloba caracasana Meisn. & Árbol & Dioico & Ornitocoria \\
\hline Ruprechtia ramiflora (Jacq.) C.A. Mey. & Árbol & Dioico & Anemocoria \\
\hline \multicolumn{4}{|l|}{ Rubiaceae } \\
\hline $\begin{array}{l}\text { Rosenbergiodendron formosum (Jacq.) Fagerl. var. } \\
\text { formosum }\end{array}$ & Arbusto & Hermafrodita & Ornitocoria \\
\hline \multicolumn{4}{|l|}{ Sapindaceae } \\
\hline Paullinia cururu L. & Trepadora & Hermafrodita & Ornitocoria \\
\hline
\end{tabular}

Referencias. ${ }^{a}$ Síndrome de dispersión principal; ${ }^{b}$ La inflorescencia también presenta flores estériles con estaminodios. 
Anexo 2. Resumen de los sistemas reproductivos de las especies inferidos con base en la relación polen/óvulo (P/O), la presencia de dicogamia y/o hercogamia, y la producción de frutos sin intervención de polinizadores (reproducción espontánea). Para cada especie es señalado además el sistema de compatibilidad descrito en la literatura.

Annex 2. Summary of the reproductive systems of the species that were inferred based on the pollen/ovule (P/O) ratio, the presence of dicogamy and/or herkogamy, and fruit production without pollinator intervention (spontaneous reproduction). The compatibility system described in the literature is also indicated for each species.

\begin{tabular}{|c|c|c|c|c|c|c|}
\hline Especie & $\begin{array}{c}\text { Relación } \\
{\mathrm{P} / \mathrm{O}^{\mathrm{a}}}\end{array}$ & Dicogamia & Hercogamia & $\begin{array}{c}\text { Reproducción } \\
\text { espontánea }\end{array}$ & $\begin{array}{l}\text { Sistema de } \\
\text { compatibilidad }\end{array}$ & $\begin{array}{c}\text { Tipo de } \\
\text { reproducción }\end{array}$ \\
\hline Ammannia auriculata & Baja & No & No & ND & $A C^{b}$ & Autogamia \\
\hline Coccoloba caracasana & ND & - & Si (dioica) & ND & - & $\begin{array}{l}\text { Información } \\
\text { insuficiente }\end{array}$ \\
\hline Copernicia tectorum & Alta & ND & No & No & ND & Xenogamia \\
\hline Cordia dentata & Alta & No & $\mathrm{Si}$ & No & $\mathrm{Al}^{\mathrm{c}, \mathrm{d}}$ & Xenogamia \\
\hline Cynophalla hastata & Alta & No & No & No & Parcialmente $\mathrm{Al}^{\mathrm{d}}$ & Xenogamia \\
\hline Eleocharis mutata & Alta & $\mathrm{Si}$ & No & No & ND & Xenogamia \\
\hline Geoffroea spinosa & Alta & No & No & No & ND & Xenogamia \\
\hline Guazuma ulmifolia & Baja & $\mathrm{Si}$ & $\mathrm{Si}$ & No & $A l^{d, e, f, g}$ & Xenogamia \\
\hline Hecatostemon completus & Alta & No & No & No & ND & Xenogamia \\
\hline Ipomoea carnea & Alta & No & No & No & Parcialmente $\mathrm{Al}^{\mathrm{h}}$ & Xenogamia \\
\hline Lemna aequinoctialis & Baja & No & No & ND & $A C^{i}$ & Autogamia \\
\hline Ludwigia erecta & Baja & No & No & ND & ND & Autogamia \\
\hline Ludwigia leptocarpa & Baja & No & No & $\mathrm{Si}$ & $A C^{J}$ & Autogamia \\
\hline Neptunia plena & Baja & ND & No & ND & ND & $\begin{array}{l}\text { Información } \\
\text { insuficiente }\end{array}$ \\
\hline $\begin{array}{l}\text { Nymphaea amazonum ssp. } \\
\text { amazonum }\end{array}$ & ND & No & No & No & ND & Xenogamia \\
\hline Nymphaea pulchella & Baja & No & No & $\mathrm{Si}$ & $A C^{k}$ & Autogamia \\
\hline Paullinia cururu & Alta & ND & No & ND & ND & $\begin{array}{l}\text { Información } \\
\text { insuficiente }\end{array}$ \\
\hline Pistia stratiotes & Baja & No & No & ND & ND & Autogamia \\
\hline Pithecellobium lanceolatum & Alta & No & No & No & ND & Xenogamia \\
\hline $\begin{array}{l}\text { Rosenbergiodendron } \\
\text { formosum var. formosum }\end{array}$ & ND & No & No & No & Parcialmente $\mathrm{Al}^{\mathrm{d}}$ & Xenogamia \\
\hline Ruprechtia ramiflora & ND & - & Si (dioica) & ND & - & $\begin{array}{l}\text { Información } \\
\text { insuficiente }\end{array}$ \\
\hline Senna bicapsularis & Alta & No & $\mathrm{Si}$ & No & ND & Xenogamia \\
\hline Sesbania exasperata & Alta & No & No & ND & ND & $\begin{array}{l}\text { Información } \\
\text { insuficiente }\end{array}$ \\
\hline Spilanthes urens & Alta & No & No & No & ND & Xenogamia \\
\hline Tillandsia flexuosa & Alta & No & $\mathrm{Si}$ & No & $\mathrm{Al}^{\mathrm{d}}$ & Xenogamia \\
\hline
\end{tabular}

Referencias. ${ }^{\mathrm{a}}$ Se consideraron bajos los valores $\mathrm{P} / \mathrm{O} \leq 400$ y altos los valores $\mathrm{P} / \mathrm{O}>400$; ${ }^{\mathrm{b}}$ Graham (2003); ${ }^{\mathrm{c}}$ Opler et al. (1975); ${ }^{d}$ N. Ramírez (datos no publ.); ${ }^{\mathrm{e}}$ Bawa (1974); ${ }^{\mathrm{f}}$ Ruíz-Zapata \& Arroyo (1978); ${ }^{\mathrm{g}}$ Bullock (1985); ${ }^{\mathrm{h}}$ LemusJiménez \& Ramírez (2005); ${ }^{\mathrm{i}}$ Landolt (1986); ${ }^{\mathrm{j}}$ Oziegbe \& Faluyi (2012); ${ }^{\mathrm{k}}$ como N. ampla en Barrios \& Ramírez (2008). 
Anexo 3. Biomasa asignada a los frutos y semillas y relación pericarpio/semilla de las especies estudiadas. Annex 3. Biomass assigned to fruits and seeds and pericarp/seed ratio of the species studied.

\begin{tabular}{|c|c|c|c|}
\hline \multirow[b]{2}{*}{ Especie } & \multicolumn{2}{|c|}{ Peso seco (mg) } & \multirow[b]{2}{*}{$\begin{array}{l}\text { Relación pericarpio/ } \\
\text { semilla }\end{array}$} \\
\hline & $\begin{array}{l}\text { Fruto } \\
(\bar{X} \pm \mathrm{DE})\end{array}$ & $\begin{array}{l}\text { Semilla } \\
(\bar{X} \pm \mathrm{DE})\end{array}$ & \\
\hline Ammannia auriculata Willd. & $12,41 \pm 2,29$ & $0,02 \pm 0,00$ & 0,60 \\
\hline Coccoloba caracasana Meisn. & $22,37 \pm 2,98$ & ND & - \\
\hline Copernicia tectorum (Kunth) Mart. & $1966,82 \pm 189,72$ & $1120,04 \pm 107,70$ & 0,75 \\
\hline Cordia dentata Poir. & $335,38 \pm 110,34$ & ND & - \\
\hline Cynophalla hastata (Jacq.) C. Presl & $1655,35 \pm 849,55$ & $85,86 \pm 26,25$ & 2,15 \\
\hline Eleocharis mutata (L.) Roem. \& Schult. & $1,94 \pm 8,11$ & $0,15 \pm 0,05$ & 11,93 \\
\hline Geoffroea spinosa Jacq. & $3550,30 \pm 632,01$ & $1387,97 \pm 252,61$ & 1,55 \\
\hline Guazuma ulmifolia Lam. & $438,90 \pm 123,04$ & $3,15 \pm 0,54$ & 3,49 \\
\hline Hecatostemon completus (Jacq.) Sleumer & $977,46 \pm 188,60$ & $7,35 \pm 2,42$ & 3,95 \\
\hline Ipomoea carnea Jacq. & ND & $92,86 \pm 15,06$ & - \\
\hline Lemna aequinoctialis Welwitsh & $0,04 \pm 0,01$ & ND & - \\
\hline Ludwigia erecta (L.) Hara & ND & $0,09 \pm 0,02$ & - \\
\hline Ludwigia leptocarpa (Nutt.) Hara & $61,72 \pm 22,27$ & $0,15 \pm 0,05$ & 0,67 \\
\hline Neptunia plena (L.) Benth. & $98,05 \pm 12,87$ & $7,02 \pm 0,59$ & 0,42 \\
\hline $\begin{array}{l}\text { Nymphaea amazonum Mart. \& Zucc. ssp. } \\
\text { amazonum }\end{array}$ & $1993,33 \pm 1117,21$ & $0,22 \pm 0,03$ & 0,03 \\
\hline Nymphaea pulchella DC. & $8060,00 \pm 4790,00$ & $0,80 \pm 0,34$ & 0,14 \\
\hline Paullinia cururu L. & $208,32 \pm 51,08$ & $49,60 \pm 11,89$ & 0,92 \\
\hline Pistia stratiotes L. & $35,31 \pm 12,20$ & $1,17 \pm 0,19$ & 0,15 \\
\hline $\begin{array}{l}\text { Pithecellobium lanceolatum (Humb. \& Bonpl. ex } \\
\text { Willd.) Benth. }\end{array}$ & $4323,27 \pm 1531,33$ & $257,12 \pm 96,88$ & 1,66 \\
\hline $\begin{array}{l}\text { Rosenbergiodendron formosum (Jacq.) Fagerl. } \\
\text { var. formosum }\end{array}$ & $758,12 \pm 223,75$ & $8,87 \pm 2,08$ & 3,60 \\
\hline Ruprechtia ramiflora (Jacq.) C.A. Mey. & ND & ND & - \\
\hline Senna bicapsularis (L.) Roxb. & $1793,34 \pm 640,50$ & $14,98 \pm 2,84$ & 1,50 \\
\hline Sesbania exasperata H.B.K. & $191,49 \pm 52,49$ & $4,25 \pm 0,53$ & 0,80 \\
\hline Spilanthes urens Jacq. & $0,39 \pm 0,12$ & $0,17 \pm 0,06$ & 1,29 \\
\hline Tillandsia flexuosa Sw. & $185,09 \pm 79,74$ & $0,91 \pm 0,28$ & 1,26 \\
\hline
\end{tabular}

Referencias. $\mathrm{DE}=$ desviación estándar, $\mathrm{ND}=$ no disponible. 